\title{
Motion Field Estimation Using U-Disparity Map in Vehicle Environment
}

\author{
Seung-Woo Seo*, Gyu-Cheol Lee* and Ji-Sang Yoo ${ }^{\dagger}$
}

\begin{abstract}
In this paper, we propose a novel motion field estimation algorithm for which a Udisparity map and forward-and-backward error removal are applied in a vehicular environment. Generally, a motion exists in an image obtained by a camera attached to a vehicle by vehicle movement; however, the obtained motion vector is inaccurate because of the surrounding environmental factors such as the illumination changes and vehicles shaking. It is, therefore, difficult to extract an accurate motion vector, especially on the road surface, due to the similarity of the adjacent-pixel values; therefore, the proposed algorithm first removes the road surface region in the obtained image by using a U-disparity map, and uses then the optical flow that represents the motion vector of the object in the remaining part of the image. The algorithm also uses a forward-backward error-removal technique to improve the motion-vector accuracy and a vehicle's movement is predicted through the application of the RANSAC (RANdom SAmple Consensus) to the previously obtained motion vectors, resulting in the generation of a motion field. Through experiment results, we show that the performance of the proposed algorithm is superior to that of an existing algorithm.
\end{abstract}

Keywords: Forward-backward error, Motion-field estimation, Optical flow, U-disparity

\section{Introduction}

Recently, many research works on self-driving cars and smart cars have been actively carried out in vehicle-related areas. Smart car refers to a future vehicle that provides safety and convenience to drivers by integrating cuttingedge technologies such as computers, communications, and sensors into the design of a vehicle; especially, a variety of research studies on lane recognition, front-obstruction detection, road-sign recognition, and pedestrian recognition using vision system are in progress. Motion field estimation, which is the key technology for above research, gives a motion vector regarding a vehicle's movement. As the camera attached to the vehicle is affected by environmental factors and vehicular movement, the possibility of a motion vector error occurrence is high; therefore, to estimate the motion field accurately, it is important to estimate vehicle's movement in consideration of the environmental factors.

Three methods can be used to estimate the vehicle's movement; a method of segmenting image, the RANSAC (RANdom SAmple Consensus) and the Kalman filter [1-4]. In the first method, the given image is segmented into three parts, an upper, a middle, and a lower and the upper and middle parts are used for estimation. It also uses a part of the image that is least affected while a vehicle moves forward or backward. The algorithm can, however, be affected by various factors such as change of viewpoints, illumination, scale, and position changes because only

$\dagger$ Corresponding Author: Dept. of Electronic Engineering, Kwangwoon University, Korea. (jsyoo@kw.ac.kr)

* Dept. of Electronic Engineering, Kwangwoon University, Korea. ( \{zlrzlr2000, gyucheo10116\}@gmail.com)

Received: January 27, 2016; Accepted: October 2, 2016 pixel information is used to estimate the motion vector.

The RANSAC is an iterative method to estimate parameters of a mathematical model from a set of observed data that contains outliers [5]. However, if there is an abnormal motion in the data, the estimation of a completely different motion occurs. While the Kalman filter [6] shows a robust performance to noise, it requires that the system of the vehicle should be known. Furthermore, the performance of the algorithm depends on the way how to choose the system model.

As such, the existing algorithms are very sensitive to the changes of viewpoint, illumination, and scale and also yield an inaccurate estimation result when noise is involved. In this paper, we therefore propose an algorithm to solve these problems. The proposed algorithm can estimate the motion field accurately with road-surface removal using a stereocamera system and forward-backward error removal using the optical flow.

The road surface removal method using a stereo camera system removes the road surface region that underpins the high possibility of a motion vector error by using the depth information. The depth information can be obtained from the utilization of an infrared-ray sensor or a radar sensor. While the cost of an infrared-ray sensor is inexpensive, though, the use of this sensor is limited in cases of short measurable distances; alternatively, the measurable distance of a radar is long and accurate but it is expensive. We therefore use a stereo camera system to obtain the depth information, where the stereo-vision algorithm is used to reconstruct the three-dimensional structure $[7,8]$.

Generally, in the region corresponding to the road surface in an image, the adjacent pixels have similar intensity value to each other, the possibility of an error 
occurrence is high in the process of obtaining the optical flow; therefore, the proposed algorithm removes the road surface areas by using a U-disparity map [9]. When applying Lukas-Kanade method $[10,11]$ to extract the motion vector, it is possible to extract an unsuitable motion vector because it uses only pixel information. Therefore, to estimate the motion vector accurately, the forwardbackward error removal method [12] is used in the proposed algorithm, and the motion field is estimated through the vehicle's movement. In this paper, we confirm that the performance of the proposed algorithm is superior to that of an existing one through experiments.

In session 2, the road surface removal algorithm with a U-disparity map is introduced. The method to obtain depth information using stereo vision system is also explained. In session 3, the forward-backward error removal and motionfield estimation are explained. In session 4, a comparison of the proposed algorithm with an existing one confirms that the performance of the proposed algorithm is superior and the conclusion is presented in session 5

\section{Road-surface Removal}

\subsection{Acquisition of depth information using a stereo- vision system}

Stereo vision is a method for extracting the disparity and depth information from the left and right images acquired by a stereo-camera system, followed by a reconstruction of the three-dimensional structure [7, 8]. After a disparity map is first generated from two images, it is used to obtain the depth map. The disparity is also used to measure the size of an object and the distance between objects.

As shown in Fig. 1, the disparity is extracted by using the principles of trigonometry in a stereo vision system. $b$ in Fig. 1 is the base line that represents the distance between the left and right cameras; $f$ is the focal length that represents the distance between the image sensor and the lens.

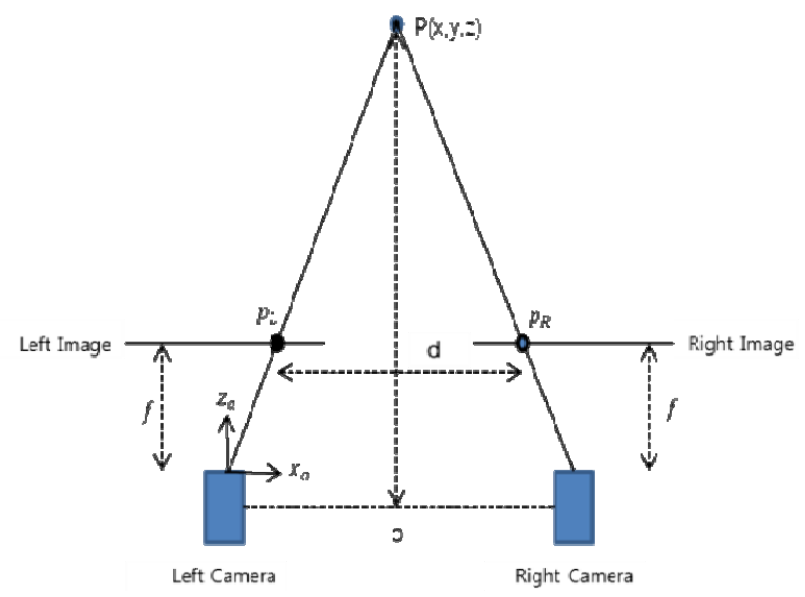

Fig. 1. Stereo vision system
In Fig. 1, $x_{a}$ and $z_{a}$ are the $\mathrm{x}$ axis and optical axis of the camera, respectively and $P$ is the $(\mathrm{x}, \mathrm{y}, \mathrm{z})$ coordinate that represents a point in the real world. When shooting $P$ with the camera, the projected point on the image plane becomes $p$. Assuming that two cameras are accurately installed on a straight line, each of the $x$-axis coordinates of projected two points, $p_{L}$ and $p_{R}$ on the left and right image planes become $f x / z$ and $(x-b) / z$, and the difference $d$ between these two points defines the disparity as $f b / z$ in this case. At this time, the depth value becomes $f b / d$ and it can be obtained by using the disparity value. The images generated by the obtained disparity value and the depth value are the disparity map and the depth map, respectively.

Due to the different characteristics of each camera's sensors and lens, additional work is required to apply the stereo-vision system; that is, a camera-calibration process is necessary to determine the inter-camera location relation. After this process, the disparity and depth values should be obtained.

\subsection{Road surface removal using U-disparity map}

When the feature points of an image are extracted in a vehicular environment and the motion vector of a corresponding feature point is directly obtained using optical flow, the possibility of an error occurrence is high for the image to include road surface region. It is difficult to obtain an accurate estimation of the motion vector by using the optical flow because, generally, the property of the adjacent pixels in the region of the road surface is

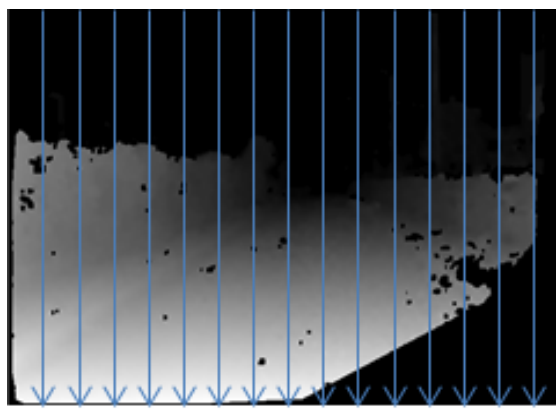

(a)

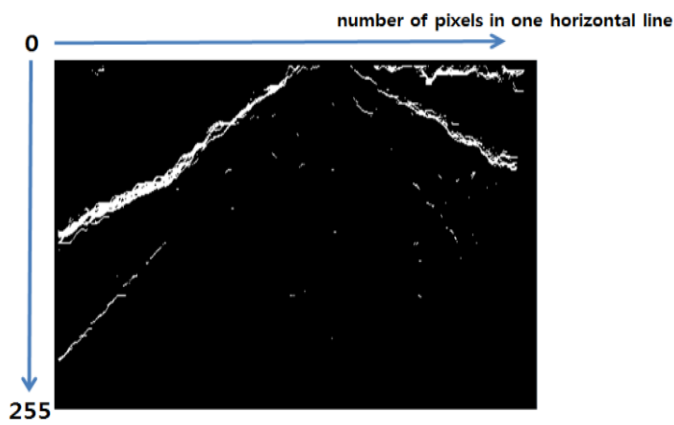

(b)

Fig. 2. (a) The process of generating U-disparity map, (b) the generated U-disparity map 


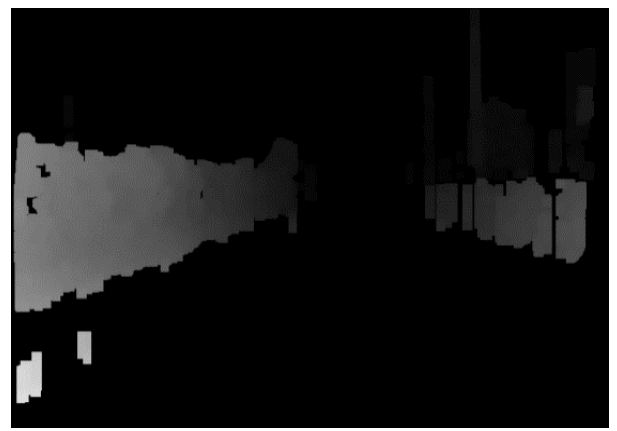

Fig. 3. Result of road surface removal using U-disparity map

similar to each other; therefore, we first try to remove the corresponding region to the road surface in an image by using the U-disparity map. [9]

A U-disparity map is a visualization of the histogram in each vertical line, whereby the number of pixels are accumulated with the same disparity value on each vertical line of the disparity map that is obtained using the stereovision system, as shown in Fig. 2(b). In Fig. 2(b), the intensity of the image is the accumulated frequency of each disparity value represented by a pixel value of 0 through 255. If the vertical-axis resolution of the image is larger than 256, the accumulated disparity value can be larger than 255; but 255 was used to represent them as an image.

The U-disparity map is used to remove the road surface area. Generally, the disparity values of pixels in each object are very similar, but the disparity values of pixels on the road region are different because the road usually faces toward the vanishing point in an image. Therefore, the region corresponding to an object is expressed as a high intensity value in a U-disparity map but the shown intensity value of the road surface is low. The proposed algorithm achieves the road-surface removal through the removal of the dark regions in the U-disparity map; Fig. 3 shows the result of road-surface-removal by the Udisparity map.

\section{Motion Field Estimation}

\subsection{Extraction of motion vectors using optical flow}

Optical flow is a pattern of the apparent motions of objects; by using it, the motion of object or the motion of the background can be identified. To extract an accurate motion vector, the optical flow is needed to find the easily identified point called the feature point regardless of the shape, size, and location changes of the object. The variety of algorithms for the feature-point extraction include SIFT, SURF, and FAST, but the most representative algorithm, Harris corner [13] is used in this paper.

Optical flow is classified into a dense one or a sparse one. The dense optical flow requires the acquisition of the motion vector from all of the pixels of an image and the representative method is Horn-Schunck.[14] The disadvantage of this algorithm, however, is a long process time.

For the sparse optical flow, the feature points are obtained in advance by using a feature-point-extraction algorithm such as Harris corner or SIFT and then the motions of the extracted feature points are estimated; Lucas-Kanade is the representative algorithm for this purpose. Regarding the sparse optical flow, even though a step for the feature-point extraction is added, its computational burden is relatively less because it finds motion only at feature points. In this paper, we use Lucas-Kanade algorithm to estimate the motion vector.

Lucas-Kanade is based on the three assumptions, brightness constancy, temporal continuity, and spatial coherence. However, the accuracy of the motion vector gets lower due to the difficulty regarding the satisfaction of all three assumptions. The possibility that the extracted motion vector is inappropriate is high because the feature point is not robust due to the similarity of the pixel values in the region, especially on the road surface; therefore, feature points in the region excluding the road surface is extracted and the optical flow is obtained in the proposed algorithm.

Fig. 4(a) shows the motion vector that has been obtained from the image, including the road surface; as this image shows the longitudinal movement of a vehicle, it should be a shape of the external motion vector spread out of the

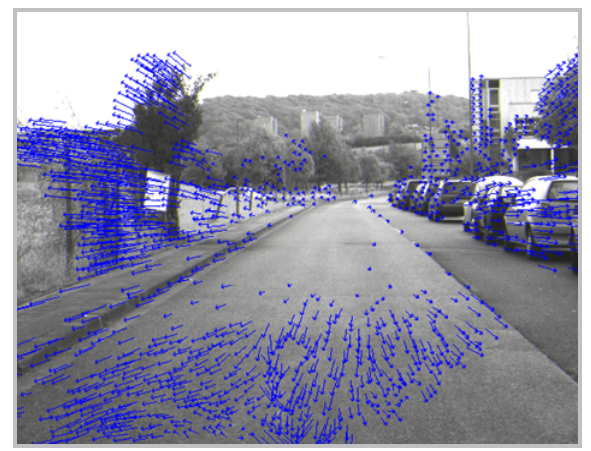

(a)

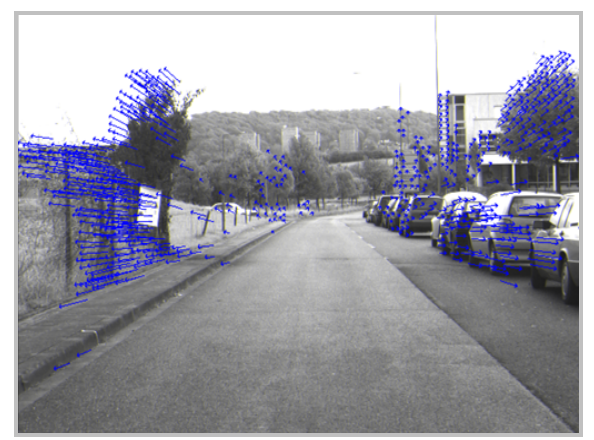

(b)

Fig. 4. Optical Flow : Optical flow, (a) with road surface (b) without road surface 
center in the image. The motion vector is inaccurate, however, as shown in Fig. 4(a) with road surface region. Fig. 4(b) shows motion vectors that have been obtained through the use of the proposed algorithm, excluding the road surface. By removing the inaccurate motion vector, the accuracies of the vehicle-motion information and the motion field can be improved.

\subsection{Forward-backward error removal}

Even though road surface region is removed and the motion vector is obtained using the Lukas-Kanade algorithm $[10,11]$, a motion-vector error still occurs due to an illumination change or a complex texture. In the proposed algorithm, we use forward-backward error removal method [12] to solve this problem.

This method finds the optical flow in both directions, forward and backward directions temporary. And then if obtained motion vectors are not the equal, they are removed.

The forward direction finds the optical flow from the $(\mathrm{t}-1)^{\text {th }}$ frame to the $\mathrm{t}^{\text {th }}$ frame, and the backward direction finds the optical flow from the $\mathrm{t}^{\text {th }}$ frame to the $(\mathrm{t}-1)^{\text {th }}$ frame.

Fig. 5 shows an example of the forward-backward optical flow. According the optical flow in forward direction, the point (1) is corresponding to the point (2) and similarly, the point (2) is corresponding to the point (1) by the optical flow in backward direction. This case is therefore considered as to be normal since the corresponding points are identical.

The point (3) and (4) are not identical even though they are the results of each optical flow in forward and backward direction. Thus this optical flow is considered not to be correct.

In Fig. 6, forward-backward error distance is defined as

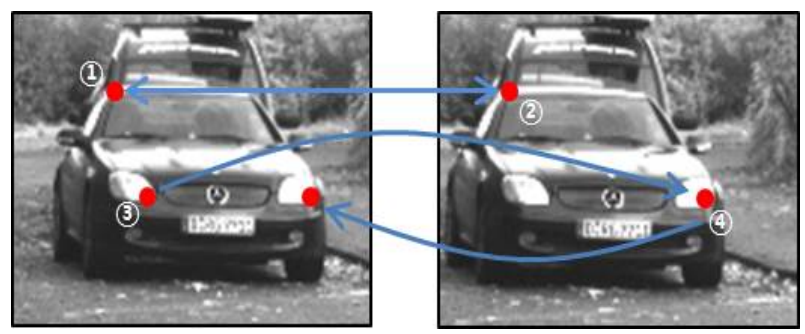

Fig. 5. Examples of forward-backward optical flow

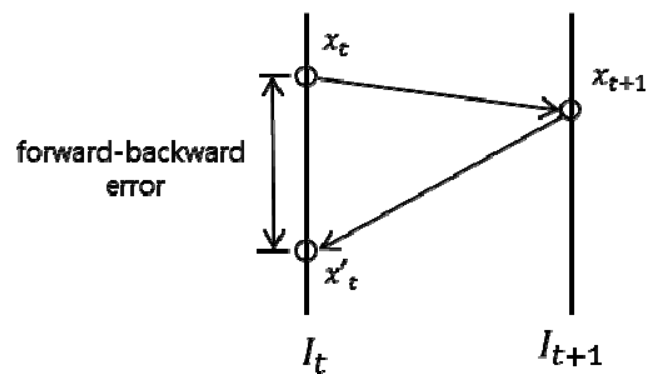

Fig. 6. Forward-backward error distance the Euclidean distance between the point $x_{t}$ and $x_{t}^{\prime}$ where $x_{t}$ is the original point in frame $I_{t}$ and $x_{t}^{\prime}$ is the corresponding point of $x_{t+1}$ in frame $I_{t+1}$ by the optical flow in backward direction.

In this paper, the valid motion vectors which have 0 value of forward-backward error distance are used for motion field estimation.

\subsection{Motion-field estimation using RANSAC}

In this paper, we generate a motion field by estimating only the camera motion from motion vectors obtained in previous step. To estimate the camera motion, we need a motion model that represents mathematical relationship between the camera motion and motion vectors

In this paper, the Longuet-Higgins equation [15] is used to define this motion model. The Longuet-Higgins equation calculates the velocity in 2-dimentional space using a camera motion and points in 3-dimentional space.

As shown in Fig. 7, the camera motion used in the Longuet-Higgins equation is composed of following two movements: translation $\left(T=\left[T_{x}, T_{y}, T_{z}\right]\right)$ and rotation (Yaw $\left(\omega_{x}\right)$, Pitch $\left(\omega_{y}\right)$, Roll $\left.\left(\omega_{z}\right)\right)$.

Assuming that the interval between $\mathrm{t}^{\text {th }}$ frame and $(\mathrm{t}+1)^{\text {th }}$ is very short, the velocity of a point in 3-dimensional space can be defined using these two movements. If the world coordinate is $(\mathrm{x}, \mathrm{y}, \mathrm{z})$, the velocity of the point in 3dimensional space is shown by Eq. (1), as follows:

$$
\begin{gathered}
V=-T-\omega \times P \\
T=\left[\begin{array}{c}
T_{x} \\
T_{y} \\
T_{z}
\end{array}\right] \omega=\left[\begin{array}{c}
\omega_{x} \\
\omega_{y} \\
\omega_{z}
\end{array}\right] P=\left[\begin{array}{c}
X_{t} \\
Y_{t} \\
Z_{t}
\end{array}\right]
\end{gathered}
$$
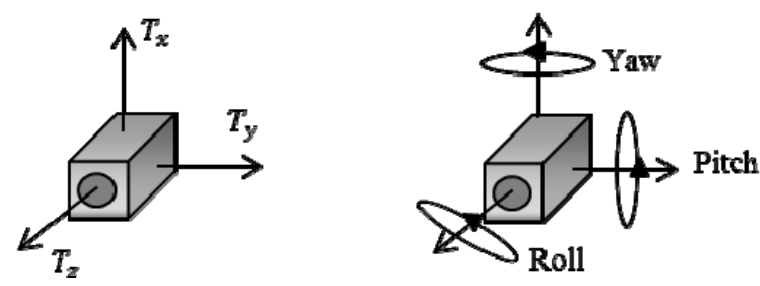

Fig. 7. Camera motion model

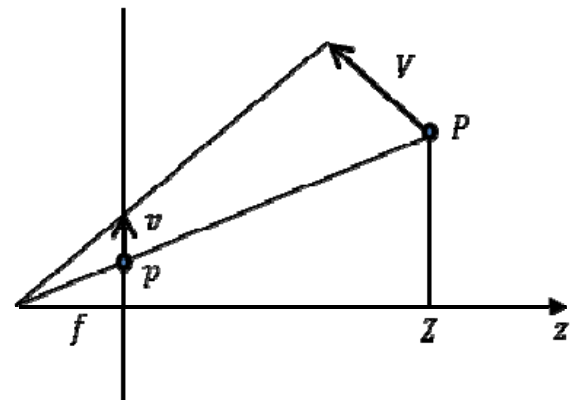

Fig. 8. Projected point from 3-dimensional space to 2 dimensional 
where $V$ is the velocity of a point in 3-dimensional space, $T$ is the linear velocity of the camera, $\omega$ is the angular velocity of the camera, and $P$ is a point in the $\mathrm{t}^{\text {th }}$ frame. $\times$ $x$ means the cross product. Since $T, T, \omega$, and $P$ are all vectors with 3 rows, they can rewritten in each component of $\mathrm{x}, \mathrm{y}$, and $\mathrm{z}$ directions by Eq. (2):

$$
\begin{gathered}
V_{x}=-T_{x}-\omega_{y} Z \times \omega_{z} Y \\
V_{y}=-T_{y}-\omega_{z} X \times \omega_{x} Z \\
V_{z}=-T_{z}-\omega_{x} Y \times \omega_{y} X
\end{gathered}
$$

Fig. 8 shows the projected point $p$ of 3-dimensional point $P$ onto two-dimensional space. The movement of point in 3-dimensional space can be projected onto 2dimensional space, and the projected point $p$ can be obtained using the proportional relation with the point $P$ as in Eq. (3)

$$
p=\frac{P}{Z} f
$$

where $f$ is the focal length of the camera.

The velocity of point in 2-dimensional space can be obtained by differentiating the point $p$ with respect to time $t$. By Eq. (3), the velocity of the point in 2-dimensional space is then expressed with parameters in 3-dimensional space as in Eq. (4).

$$
\begin{aligned}
\frac{d P}{d t} & =v=\frac{d \frac{f P}{Z}}{d t}=\frac{f}{Z^{2}}\left[\frac{d P}{d t} Z-P \frac{d Z}{d t}\right] \\
& =\frac{f}{Z^{2}}\left[V Z-P V_{z}\right]=f \frac{V}{Z}-P \frac{V_{z}}{Z}
\end{aligned}
$$

Eq. (5) represents the relationship between the camera motion, motion vectors and the two-dimensional velocity by substituting Eq. (2) for (4).

$$
\begin{aligned}
& v_{x}=\frac{T_{z} x-T_{x} f}{Z}-\omega_{y} f+\omega_{z} f+\frac{\omega_{x} x y}{f}-\frac{\omega_{y} x^{2}}{f} \\
& v_{y}=\frac{T_{z} y-T_{y} f}{Z}-\omega_{x} f+\omega_{z} x+\frac{\omega_{y} x y}{f}-\frac{\omega_{x} y^{2}}{f}
\end{aligned}
$$

where $v=\left[v_{x}, v_{y}\right]$ is the 2-dimensional velocity of a point, $T=\left[T_{x}, T_{y}, T_{z}\right]$ is the linear velocity of the camera, $\omega=\left[\omega_{x}, \omega_{y}, \omega_{z}\right]$ is the angular velocity of the camera, $f$ is the focal length of the camera, and $x, y$ is the coordinate of two-dimensional space.

To estimate a precise camera motion, outliers that are incorrect motion vectors should be removed. In the proposed algorithm, the RANSAC is used to remove outliers. Camera motions, $T$ and $\omega$ can be obtained by applying RANSAC to the motion model and finally the motion vector $\left[v_{x}, v_{y}\right]$ in an image is obtained by Eq. (5) with the camera motions, $T$ and $\omega$.

\section{Experiments}

The proposed algorithm is implemented by Microsoft Visual Studio C++ 2010 and OpenCV 2.4.8 library with Intel's i5-2500 3.30 dual core CPU and 8 GB of memory. We used Daimler Pedestrian Dataset [16] which is stereo dataset with $640 \times 480$ resolution as test data.

As mentioned already, there are two types of optical flows: dense optical flow and sparse optical flow. While the accuracy of the dense optical flow is more precise than that of the sparse optical flow, the processing time for the dense optical flow is longer.

Table 1 shows the processing time of both the dense optical flow and the sparse optical flow. On average, the dense optical flow takes 0.34 second per frame, while the sparse one takes 0.069 second. The sparse optical flow was therefore adopted for the proposed algorithm because of the fast processing time.

However, since the sparse optical flow has lower precision, using both forward-backward error removal and road surface removal algorithms, we have to reduce the amount of errors in motion vectors.

Fig. 9 shows the obtained motion vectors and the estimated motion fields by using only the optical flow and the proposed algorithm, respectively. Fig. 9(a) is obtained motion vectors only using optical flow and its accuracy tends to be lower at road surface region as you can see.

Table 1. Processing time per frame for the optical flow

\begin{tabular}{c|c|c}
\hline & Dense optical flow & Sparse optical flow \\
\hline Processing time per frame & $0.34 \mathrm{~s}$ & $0.069 \mathrm{~s}$ \\
\hline
\end{tabular}

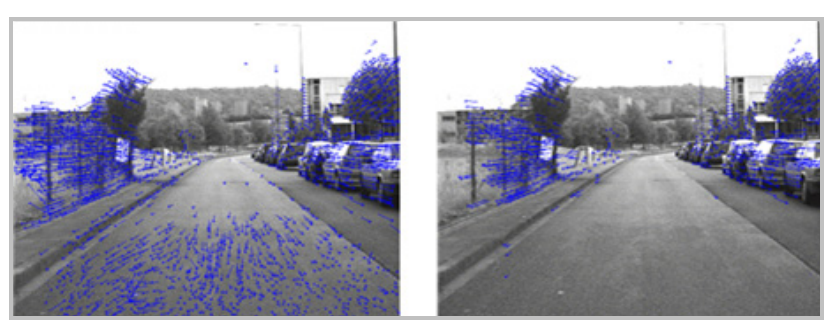

(a)

(b)

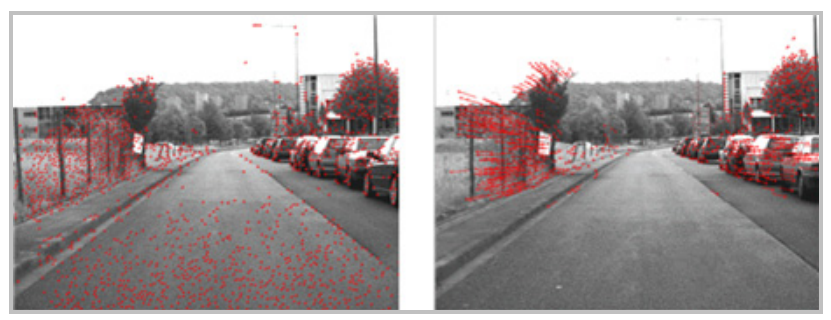

(c)

(d)

Fig. 9. Motion vectors obtained by (a) only optical flow (b) by the proposed algorithm, and motion fields estimated (c) with (a), (d) with (b) 
Thus, it is not appropriate to use these motion vectors to estimate a camera motion. Fig. 9(c) is estimated motion field using motion vectors obtained in Fig. 9(a).

Test image of Fig. 9 contains longitudinal movement of a vehicle and thus its motion vector should have radial patterns. However, estimated motion vectors are different from actual camera motion due to wrong estimated motions as in Fig. 9(c). Fig. 9(b) is motion vectors obtained by the proposed algorithm. In the proposed algorithm, both the forward-backward removal and road surface removal algorithms are used to enhance the accuracy of motion vectors. It is shown that the proposed algorithm resulted in more precise motion field of Fig. 9 (d).

Fig. 10 shows the estimated motion field of the image including some objects. In the case where moving object exists in the image, an error could occur in the motion field estimation process due to the motion of moving objects. Fig. 10 (a) is the estimated motion field of the image contains an moving object only using the optical flow and Fig. 10 (b) is the estimated motion field of the same image using the proposed algorithm. It is shown that the motion fields around tree or car region is correctly estimated.

Fig. 11 shows the result of the performance comparison between the proposed algorithm and a conventional algorithm, where the given image is segmented into three parts, an upper, a middle, and a lower and only the upper and middle parts are used for estimation. Consequently,

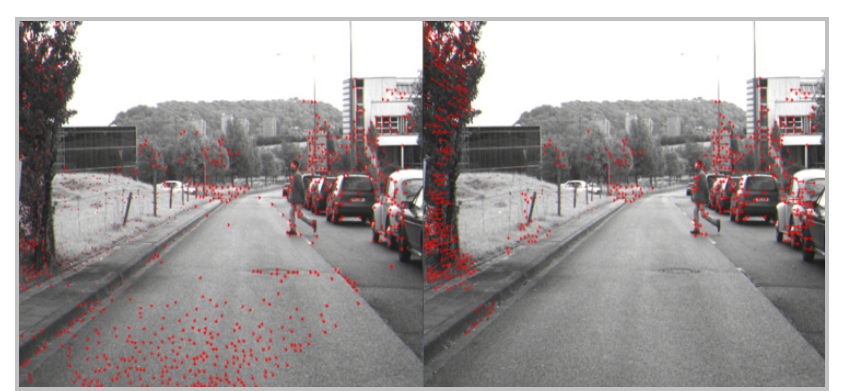

(a)

(b)

Fig. 10. Motion fields estimated (a) by only optical flow and (b) the proposed algorithm with a human being as an object

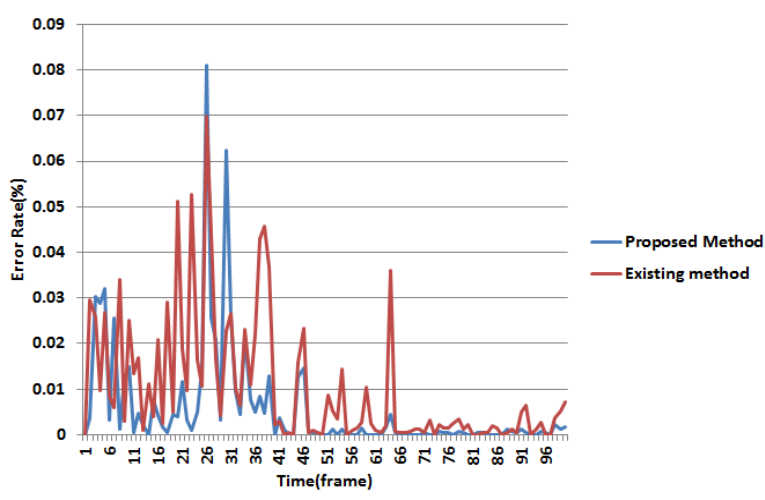

Fig. 11. Comparison of error rate when there exist incorrect motion vectors in upper and middle parts it results in unprecise motion field.

The $x$ axis in Fig. 11 shows temporal frames, while the $y$ axis shows the error rate between the ground truth motion field and estimated motion field using each algorithm. Ground truth motion field is generated using yaw rates.

The higher error rate value on $y$ axis in the graph, represents the lower accuracies of the estimated motion field. Thus, as a result of the performance comparison between the proposed and existing algorithms, it is possible to confirm that the error rate of the proposed algorithm is lower than that of the existing one in almost every frame.

Table 2 shows results of a comparison between the average error rates of the existing and proposed algorithms. While the existing algorithm shows an average error rate of 0.028 , the average error rate of the proposed algorithm is 0.005 . This indicates proposed algorithm produces 5.6 times better result than the existing algorithm.

Fig. 12 shows the comparison of yaw rates between the proposed and existing algorithms. The yaw rate shows the degree of the vehicle rotation toward the yaw direction; therefore, the estimation accuracy can be measured by comparing the given data and the ground truth. Fig. 12 (a) shows the result of the comparison between yaw rates of

Table 2. Comparison of average error rate

\begin{tabular}{c|c|c}
\hline & Existing algorithm [1] & Proposed algorithm \\
\hline Average error rate & 0.028 & 0.005 \\
\hline
\end{tabular}

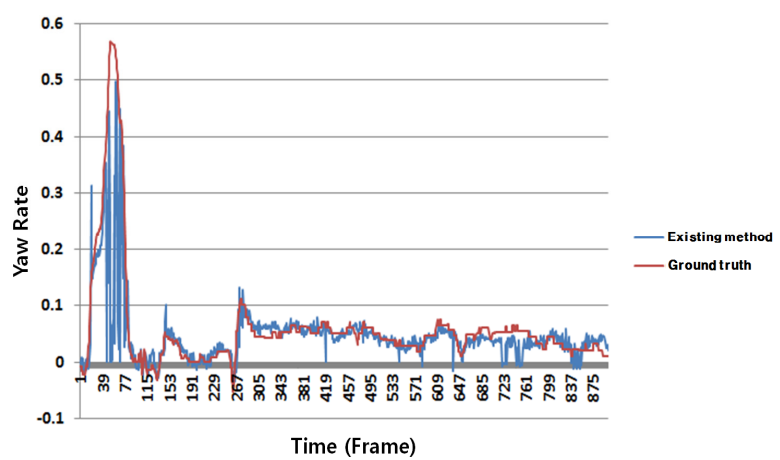

(a)

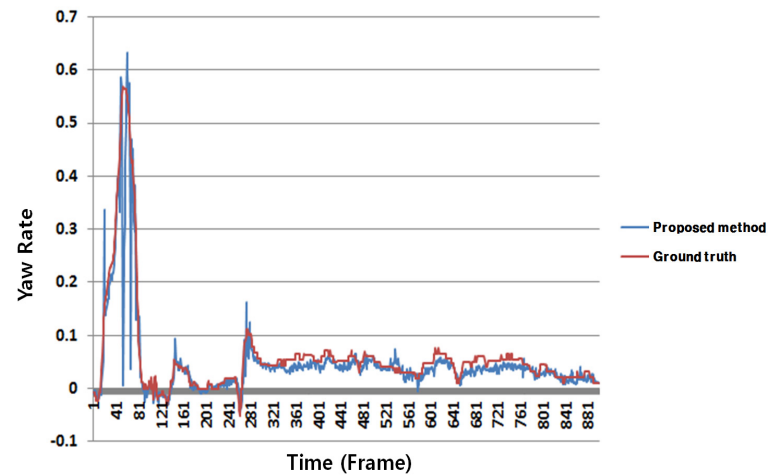

(b)

Fig. 12. Comparison of yaw rate 
the existing algorithm and the ground truth. The part with the high yaw rate in the vicinity of the $50^{\text {th }}$ frame means that there exists rotational movement of a vehicle, and the part with a steady yaw rate after the $80^{\text {th }}$ frame means that there exists only the longitudinal movement of a vehicle. As you can see, the existing algorithm yields poor results in the yaw rate estimation around the rotational movement. Also, error occurred during the longitudinal motion.

Fig. 12 (b) shows the result of the comparison between the yaw rates of the proposed algorithm and the ground truth. The proposed algorithm is more accurate than that of the existing one; furthermore, it confirms that even minor errors of estimation were removed during the longitudinal movement.

\section{Conclusion}

In this paper, we proposed a motion field estimation algorithm though a U-disparity map and forward-backward error removal method. Since extraction of motion vectors in a vehicular environment usually causes some errors especially, in the road surface regions or background, poor motion field estimation is, thus resulted in. To solve this problem, we proposed a novel motion field estimation algorithm for which a U-disparity map and forward-andbackward error removal were applied in a vehicular environment

In the proposed algorithm, the motion-vector error is minimized, and a more accurate motion field can be estimated with the RANSAC. Through experiments, we showed that the motion-vector errors were reduced, and the accuracy of the proposed algorithm was definitely higher than that of the existing one.

\section{Acknowledgements}

This work was supported by an Institute for Information \& Communications Technology Promotion (IITP) grant funded by the Korea government (MSIP) (No. R0132-161005), Content visual browsing technology in the online and offline environments).

\section{References}

[1] K. Yamaguchi, T. Kato and Y. Ninomiya, "Vehicle ego-motion estimation and moving object detection using a monocular camera", in Proc. Int. Conf. Pattern Recognition, Hong Kong, pp. 610-613, 2006

[2] O. Pink, F. Moosmann and A. Bachmann, "visual features for vehicle localization and ego-motion estimation", in Proc. IEEE Intell. Veh. Symp, Xi'an, pp. 254-260, 2009
[3] G. Ligorio and A. M. Sabatini, "Extended kalman filter-based methods for pose estimation using visual, inertial and magnetic sensors: comparative analysis and performance evaluation", Sensors, vol. 13, no. 2, pp. 1919-1941, Feb. 2013

[4] F. J. Sharifi and M. Marey, "A kalman-filter-based method for pose estimation in visual servoing", IEEE Trans. Robotics, vol. 26, no. 5, pp. 939-947, Oct. 2010

[5] M. A. Flschier and R. C. Bolles, "Random sample consensus: a paradigm for model fitting with application to image analysis and automated cartography", Commun. ACM, vol. 24, no. 6, pp. 381-395, Jun. 1981

[6] V. Lippiello, B. Siciliano and L. Villani, “Adaptive extended kalman filtering for visual motion estimation of 3D objects", Control Eng. Practice, vol. 15, no. 1, pp.123-134, Jan. 2007

[7] W. Jang, C Lee and Y. Ho, "Efficient depth map generation for various stereo camera arrangements", J. KICS, vol. 37, no. 6, pp. 458-463, Jun. 2012

[8] E. Baek and Y. Ho, "Stereo image composition using poisson object editing", J. KICS, vol. 39, no. 8, pp. 453-458, Aug. 2014

[9] Z. Hu and K. Uchimura, "U_V Disparity, an efficient algorithm for stereo vision based scene analysis", IEEE Intell. Veh. Symp., Las Vegas, pp. 48-54, 2005

[10] B. D. Lucas and T. Kanade, "An iterative image registration technique with an application to stereo vision", in Proc. Int. Joint Conf. Artificial Intell., Vancouver, pp. 674-679, 1981

[11] C. Song and J. Lee, "Detection of illegal u-turn vehicles by optical flow analysis", J. KICS, vol. 39, no. 10 , pp. $948-956$, Oct. 2014

[12] Z. Kalal, K. Mikolajczyk and J. Matas, "Forwardbackward error: automatic detection of tracking failures", in Proc. Int. Conf. Pattern Recognition, Istanbul, pp. 23-26, 2010

[13] C. Harris and M. Stephens, "A combined corner and edge detector", in Proc. Alvey Vision Conf., pp. 147151, Manchester, 1988

[14] B. K. P. Horn and B. Schunck, "Determining optical flow”, Artificial Intell., vol. 17, no. 1-3, pp. 185-203, Aug. 1981

[15] H. C. Longuet-Higgins and K. Prazdny, "The interpretation of a moving retinal image", The Royal Soc. London B, vol. 208, no. 1173, pp. 385-397, Jul. 1980

[16] C. Keller, M. Enzweiler and D. M. Gavila, "A new benchmark for stereo-based pedestrian detection", in Proc. IEEE Intell. Veh. Symp., Baden-Baden, 2011

[17] S. Seo, G. Lee and S. Lee, "Motion field estimation using u-disparity map and forward-backward error removal in vehicle environment", J. KICS, vol. 40, no. 12, pp. 2343-2352, Dec. 2015 


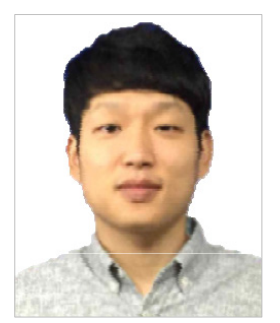

Seung-Woo Seo He received B.S degree in Electronic engineering from Kwangwoon University. His research interests are object detection, image processing and machine learning.

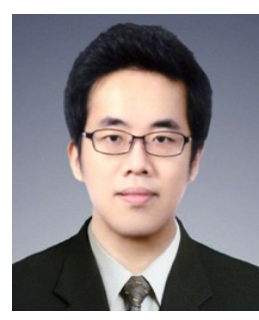

Gyu-Cheol Lee He received the B.S. and M.S. degrees from Kwangwoon University, Seoul, Korea in 2013 and 2015, all in electronics engineering. $\mathrm{He}$ is currently a candidate for a $\mathrm{PhD}$ from Kwangwoon University. His research interests are in digital image processing, multi-view synthesis, object detection and deep learning.

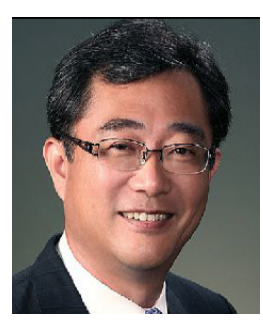

Ji-Sang Yoo He was born in Seoul, Korea in 1962. He received the B.S. and M.S. degrees from Seoul national university, Seoul, Korea in 1985 and 1987, all in electronics engineering, and Ph.D. degree from Purdue University, West Lafayette, IN, USA, in electrical engineering in 1993, respectively. From September 1993 to august 1994, he worked as a senior research engineer in industrial electronics R\&D center at Hyundai Electronics Industries Co., Ltd, Ichon, Korea, in the area of image compression and HDTV. He is currently a professor with the department of electronics engineering, Kwangwoon University, Seoul, Korea. His research interests are in signal and image processing, deep learning, and computer vision. 